\title{
Multilevel Research Strategies and Biological Systems
}

\section{Authors and affiliations}

Maureen A. O’Malley, University of Sydney ${ }^{\ddagger \dagger}$

Ingo Brigandt, University of Alberta ${ }^{\ddagger}$

Alan C. Love, University of Minnesota ${ }^{\ddagger}$

John W. Crawford, University of Sydney

Jack A. Gilbert, University of Chicago/Argonne National Laboratory

Rob Knight, University of Colorado (Boulder)

Sandra D. Mitchell, University of Pittsburgh

Forest Rohwer, San Diego State University

\section{Corresponding authors}

Maureen A. O’Malley

Ingo Brigandt

Alan C. Love maureen.omalley@sydney.edu.au

brigandt@ualberta.ca

aclove@umn.edu

\begin{abstract}
Multilevel research strategies characterize contemporary molecular inquiry into biological systems. We outline conceptual, methodological and explanatory dimensions of these multilevel strategies in microbial ecology, systems biology, protein research, and developmental biology. This review of emerging lines of inquiry in these fields suggests that multilevel research in molecular life sciences has significant implications for philosophical understandings of explanation, modelling, and representation.
\end{abstract}

\section{Acknowledgements}

We thank Carol Cleland for expertly chairing the PSA 2012 symposium that was the basis for this paper. We are grateful to the audience at that same symposium for its questions, and to Andrea Woody for comments on an earlier draft of this article.

\footnotetext{
${ }^{\dagger}$ Symposium organizer.

${ }^{\ddagger}$ These authors contributed equally as first authors to the manuscript.
} 


\section{Introduction}

For the past forty years, philosophical discussions of molecular biology have typically associated it with reductionism (Brigandt and Love 2012a). However, this framing of molecular methods and explanatory models does not do justice to much contemporary life science research (Bechtel 2010; Craver 2007; Craver and Darden 2013; Darden 2005), where a wealth of data has made it possible to derive insights into the nature and consequences of molecular function across different timescales and multiple organizational levels of biological systems. Although a major driver of systemoriented research is the availability of copious molecular data from high-throughput technologies, generating biological knowledge from it is neither straightforward nor guaranteed. An integration of different datasets, methods and models is necessary in order to understand how any particular biological system functions over time (O’Malley and Soyer 2012; see also Brigandt 2013b). In this paper we describe different types of multilevel systems that should be on the radar of philosophers of science: macromolecular complexes within cells, organisms as developing individuals, and communities composed of microbial and multicellular taxa, as well as the biogeochemical cycles in which these communities participate. The diversity and heterogeneity of investigative approaches to these multilevel systems call out for more detailed analyses in order to comprehend how scientists successfully manipulate, explain, and predict in ways that diverge from standard philosophical expectations (e.g., reductionism).

Multilevel research can be understood in a number of philosophically interesting ways. Our review begins with the innovative molecular tools being used to examine different phenomena in the life sciences, and provides a survey of several areas in which these multilevel research strategies have been deployed. We have consciously chosen systems at disparate scales in order to expose a wide range of epistemic issues that are ripe for philosophical exploration. Our aim is to draw attention to three different dimensions of inquiry - conceptual, methodological, and explanatory - that involve multiple lines of evidence and require a combination of approaches to produce integrated knowledge of biological function for these systems. There are conceptual dimensions because the systems range over multiple biological scales and require diverse units of analysis; methodological dimensions because this conceptual heterogeneity necessitates approaches that are capable of addressing multilevel composition and dynamics; and explanatory dimensions due to different activities and entities being causally connected in multilevel systems, and thus requiring explanatory models able to capture these interactions.

Even though multilevel strategies are widespread in contemporary life science, philosophers of science currently lack an understanding of how researchers use these tactics to successfully investigate dynamic systems. Traditional philosophical frameworks, such as reduction or unification, do not capture how various local epistemic considerations organize interdisciplinary research. In addition to multilevel explanation, which has been treated in some accounts of molecular mechanisms (Bechtel 2010; Craver 2007; Darden 2005), our discussion of dynamic systems also highlights philosophical issues pertaining to modelling and representation, in particular the scientific strategy of selectively approaching complexity with multiple, partial representations. 


\section{Complex Multilevel Systems}

Contemporary biologists seek to understand the complex dynamics of biological systems that are composed of multiple clusters of spatial and temporal organization. For example, in the ecological study of diverse microbial communities and ecosystems, advances in sequencing technology and computational methods (known as "metagenomic" tools) have enabled the collection and analysis of vast amounts of data about DNA, RNA, proteins, and metabolites (VerBerkmoes et al. 2009; Turnbaugh and Gordon 2008; Riesenfeld et al. 2004). Biologists use these data to manipulate, explain and predict the causal dynamics of microbial assemblages to answer a variety of research questions, such as how these assemblages are crucial to the function of larger systems in which they are embedded, from human health to planetary biogeochemistry (Dinsdale et al. 2009; Raes and Bork 2008). Whereas earlier studies concentrated on cataloguing microscopic biodiversity from "simple" extreme environments to complex soil ecologies (e.g., Tyson et al. 2004; Rondon et al. 2000), recent research attempts to characterize the functions of groups of organisms from evolutionarily diverse taxa, and the interactive dynamics of these communities over time (DeLong 2009). The process of moving from molecular inventories to a functional understanding of biological systems is challenging because many thousands of causal interactions across many levels of organization are involved. This shift to a multilevel perspective in light of a plethora of molecular data has stimulated novel approaches to traditional problems.

\section{Humans as Ecosystems}

Microbial ecologists use metagenomic tools to establish what genes and organisms inhabit specified environments. The term "microbiota" refers to the microbial consortia found in an environment, and "microbiome" refers to the community's assemblage of genes. ${ }^{1}$ While the human body is often conceptualized as an individual organism, from a systems-ecological perspective it is seen as a dynamic ecosystem of host resources and commensal microbes (Ley et al. 2006). Molecular knowledge of human microbiota has transformed investigative practices, as well as numerous aspects of human biology and medicine. A common approach is to identify microbes in various locations of the human body (e.g., skin, mouth, and large intestine) using standard genetic markers (McDonald et al. 2013; Turnbaugh et al. 2009). Another approach is to gather sequence information about the entire community genome in order to derive insights about metabolic activities or other environmental interactions (Larsen et al. 2011; Fierer et al. 2012b). Both approaches have limitations in how they identify biodiversity due to sampling, sequencing and analysis issues, but have led to numerous striking discoveries.

One unexpected finding was the uniqueness of the microbiome in each human gut. Despite the apparent similarity of much human physiology, variations in the colonization and maintenance process make individual human microbiomes

\footnotetext{
${ }^{1}$ Occasionally microbiome is used to mean microbiota (the assemblage of organisms), probably because "biome" normally means a biological community in a particular habitat. However, the "ome" in microbiome refers to "omic" data extracted from the microbiota (Gordon 2012).
} 
compositionally distinct (Ursell et al. 2012). However, inter-individual differences in these microbial communities (discerned from the abundance of species-specific marker genes) often have little or no causal impact on the host because of functional redundancy (Shade and Handelsman 2012). The composition of taxa can vary enormously but the community still performs the same functions, such as substrate metabolism or antibiotic resistance (Hamady and Knight 2009). But community composition at the phylum rather than species level can have important causal effects (Turnbaugh et al. 2009), which challenges the tenet that phyla are not "real" taxonomic groups (unlike species), nor ones with causal powers (Philippot et al. 2010). Although attributions of community or phylum causality are usually based on strong correlations, these inferences are increasingly corroborated by experimental studies, such as germ-free mice subjected to controlled microbiota transplants (e.g., from obese or lean organisms) or mice "humanized" by diet and other physiological regimes (Smith et al. 2013; Faith et al. 2011). These studies support phylum-level effects of microbiota differences on metabolic syndromes like obesity.

Human microbiome studies are multilevel in several ways. Conceptually, they interpret causal pathways within and across different kinds of systems based on the research question in view: an ecosystem of host plus microbial community, a community with functional properties (the microbiota), a molecular system (the microbiome) that represents the community, or networks of functional interactions central to that community. Methodologically, microbiome research synthesizes molecular analyses of networks with experimental methods that target populations of organisms or communities, thereby spanning a variety of system "levels" (Raes and Bork 2008). Community assembly of the microbiota is modelled either by importing classical ecological models into microbial ecology (e.g., assembly rules based on counts of taxa: Fierer et al. 2012a) or by network methods that have been developed within genomics and metagenomics (e.g., Klitgord and Segrè 2011).

\section{Global Marine Communities}

The scope of metagenomic approaches goes beyond the organismal level (e.g., the human body, occupied by multiple microbial communities) to track causal pathways with planet-wide effects. Marine environments were once thought of as sparsely inhabited deserts with linear trophic interactions that had limited roles for microbes (e.g., Lindeman 1942). This view began to change dramatically with new marine food web models that added a quantitatively significant "microbial loop" of primary producers (very small photosynthesizers) and bacterial carbon consumers (Pomeroy 1974; Azam et al. 1983). Metagenomics and microbial ecology have further transformed trophic modelling by showing that "trophic levels" are much better conceptualized as nodes that link a variety of interactions (Azam and Malfatti 2007). Microbial metabolic networks interact with the Earth's geochemistry to create biogeochemical systems that sustain and constrain life on this planet via cycles such as carbon, oxygen and nitrogen (Falkowski et al. 2008).

Molecular data are also used in analyses of biological systems that apply algorithms to datasets generated by global projects, such as the Earth Microbiome Project (http://www.earthmicrobiome.org/). These algorithms pick out significant patterns for which explanations are then sought. This is sometimes characterized as a "bottom-up" perspective in which patterns are investigated further by modelling, experiment or other analytical techniques (Gilbert et al. 2012; Faust and Raes 2012). “Top-down” 
approaches, in which a theoretical framework is applied to diverse data in order to examine the theory's applicability, are required as well (Burke et al. 2011). ${ }^{2}$ These different approaches capture many of the causal dynamics that operate in these systems at different timescales (sampling intervals, daily cycles, seasons, evolution). While many methodological challenges prevent the straightforward connection of regional systems operating on diverse scales, successful exemplars act as templates for ambitious syntheses of complicated spatial and temporal organization (e.g., Larsen et al. 2012).

Rather than culminating in measures of organismal or species-level biodiversity, the target of microbial ecological analysis is the functional capacity of communities in environments. There is a long history of conceptualizing plant and animal communities as aggregates of individual organisms, but microbial ecologists often attribute causal properties to microbial communities (Zarraonaindia et al. 2013); they are modelled as causally efficacious entities (Sterelny 2006). New data on community properties help to underwrite claims about community-level evolutionary selection due to the cohesiveness, reproducibility, and heritable properties of communities (e.g., Dethlefsen and Relman 2011).

\section{Explaining Extraordinary Viral Abundance}

Despite the growing attention to global systems of microbial communities, viral dynamics are often ignored. Although not usually considered "alive”, tiny viruses have enormous biological effects. There are more viruses than organisms, and they encode most of the genetic (sequence) diversity that exists on the planet (Rohwer and Thurber 2009). Viruses control the abundance of microbes (by killing them) and thereby regulate all of life. They also function as genetic repositories that shuttle genes back and forth between organisms (Dinsdale et al. 2009). The abundance and diversity of environmental viral sequences indicate that viruses are major drivers of global biogeochemistry alongside their traditional roles as etiological agents of diseases (Suttle 2005). A disconcerting corollary is that the totality of living organisms can be reconceptualized as a viral incubator-a controlled environment for their nurture and propagation. Viral metagenomics suggests this view is a useful representation for some research purposes, including answering questions such as why so many viruses exist on Earth.

Diverse molecular data about the viral world can be integrated by applying different conceptual and theoretical frameworks. One top-down approach is to start with the unique capacity of viruses to be transmitted as "information only" - the DNA or RNA genome alone is transmitted and can evolve, not the machinery for its replication and transmission. Viral replication is therefore unlike other types of reproduction in which offspring are formed by parents (e.g., cell division or sexual reproduction). From a "viruses as information" theoretical perspective, viral abundance can be explained as a tradeoff between informational replication and energy efficiency (Rohwer and Barott 2013). This principle can be integrated into an ecosystem model that combines other salient ecological rules (e.g., niche creation, niche exclusion, competitive dynamics) and explains microbial evolution. By

\footnotetext{
${ }^{2}$ For a different but relevant interpretation of "top down” and "bottom up" in these investigative contexts, see Zengler and Palsson (2012).
} 
focusing on informational propagation, an understanding of the role of viruses as molecular manipulators of biogeochemistry and evolutionary change at multiple levels begins to emerge.

Top-down theorizing works as a multilevel approach by integrating diverse and previously disconnected datasets pertaining to different phenomena, thereby explaining the abundance of viruses in an ecological context (Edwards and Rohwer 2005). While viral interactions must be incorporated to understand the full dynamics of any biological system, one might object that "viruses as information” captures only limited types and consequences of these interactions. But this objection assumes the goal of a single, comprehensive representation, instead of recognizing (as many scientists do) that all models are partial representations focused on some but not all features of the target phenomena. From the vantage point of energy efficiency, the "viruses as information" model makes testable and non-trivial claims about viral properties and the multilevel systems in which they are embedded. These properties are represented within a dynamic global system and interact in various ways with other biological systems (Suttle 2005). Further molecular data can be used to test and refine these claims across spatial levels and temporal scales.

\section{Multilevel Microbial Ecology}

The above illustrations from microbial ecology indicate that multilevel systems are not understood exclusively as hierarchically "nested" inside one another and that levels of spatial organization are not sufficient to conceptualize these systems. Many relevant relationships are causal (i.e., functional) rather than compositional (Hüttemann and Love 2011). When the human body is treated as an ecosystem, a human can be viewed as an interactive niche generating molecules that regulate the microbiota, while human functions - including brain activities and behaviour-are simultaneously being regulated by the microbiota (Cryan and Dinan 2012). Despite the large bodies of molecular data available to microbial ecology, the causal relationships it dissects occur in localized hierarchies of function and structure that do not presume a total representation (Love 2012). Selective aspects of target systems are examined and represented in multiple models (Weisberg 2013; Green 2013), with various types of abstraction and idealization affecting data procurement and analysis as much as experimentation and explanatory modelling.

\section{Selectivity in Multilevel Biological Research}

Any approach must choose specific system properties on which it will focus. For biological systems, physical properties also can be included. Ecosystems, for example, have abiotic as well as biotic components and dynamics. But when probing interactions in a physico-chemical system, such as soil and the biota that inhabit it, a reconceptualization of the physical properties is necessary. Instead of depicting soil as a mere aggregation of particles, it can be understood effectively as a self-organizing adaptive system (Crawford et al. 2005). Functional interdependencies between physical, chemical and biological properties must be modelled to capture interactions within and between system variables represented by different compositional relations and temporal scales. 
As the interactions between microscopic and subsurface biological agents in soil are better understood, we can comprehend their dynamic effects on macroscopic aboveground organisms, such as the impact of soil microbes on soil structure and plant growth (Young et al. 2008; Crawford et al. 2005). This array of insights afforded by molecular and mathematical analyses can be extended to additional system-states because soil ecosystems are parts of broader social systems, such as agriculture. Soil use (including damage) must be incorporated into models that attempt to predict the effects of particular agricultural strategies or climate change (Griffiths and Philippot 2011). The coordinated use of multiple datasets and models that represent systems at a variety of spatial and temporal scales challenges traditional philosophical interpretations of scientific research as relying on a "primary" representational scheme or constructing a theory of a single, focal level of organization.

Any approach, no matter its scope and capacity to coordinate different representations, is subject to limitations on the possible physical and biological interactions that can be investigated. The implication is that researchers always have to be selective. One way to embrace this challenge and pursue associated multilevel insights is through a strategy that might be called "emergent parsimony". Understanding emergence is sometimes thought to involve encompassing more parameters and data of the complex system under inquiry. Not only is this impractical, but it is also unwarranted, even in an era of expanded computational capacity. Building more of everything into the model does not automatically augment knowledge, and can in fact obscure the situation by including details that are not relevant for producing the system property that is a particular project's focus. Apparently emergent phenomena may be produced by complex interactions, but understanding them requires epistemic restraint and informed decisions about what to include and what to leave out in representational models.

Methodological and representational strategies that are sensitive to this constraint include the use and development of models that selectively circumscribe the number of variables to be measured, or which partition systems according to different compositional and temporal principles, while still being able to manipulate, predict or explain the phenomenon being investigated. Even in complex simulations that aim to include the interactions of every individual in a system (e.g., agent-based models), some parameters and expectations are built into the model to simplify its construction and make simulation feasible (Grimm 1999; Railsback 2001). Other system-oriented approaches ignore individual interactions altogether and focus on population-level variables to explain the existence and dynamics of various properties that in combination account for system function.

\section{Integrative Explanations: Mechanistic and Mathematical Models}

A characteristic feature of systems approaches in biology is the use of mathematical models and quantified molecular data to explore biological dynamics (e.g., Karr et al. 2012). Various types of models are used, but differential equations play a key role in representing and predicting a system's behaviour across time. These models were once less common in molecular biology, where causal explanations of mechanisms 
are often framed in terms of spatial relations and qualitative interactions among components. Philosophical discussion of molecular biology over the past decade has concentrated on this mechanistic explanatory strategy (Craver 2007; Darden 2005). But integrating multilevel mechanistic and mathematical models is crucial for a robust understanding of the dynamical aspects of biological systems, such as the effects of complex feedback loops or the generation of new kinds of entities and functions (Bechtel 2011, 2012; Brigandt 2013c, in press; Hüttemann and Love 2011; Mitchell 2003, 2009).

Systems biology, which focuses on intracellular networks of dynamic interactions (Krohs and Callebaut 2007), is an approach founded on the combination of mechanistic and mathematical types of models (Boogerd et al. 2007; Klipp et al. 2010; O’Malley and Soyer 2012). Computational analysis and mathematical modelling complement the experimental study of molecular entities and their causal interactions (e.g., network models applied to large molecular data sets obtained from functional genomics projects; see Karr et al. 2012). An instructive case is the segmentation clock in vertebrates (Brigandt 2013c). In early development, vertebrate segments differentiate sequentially in the embryo from head to tail. A crucial component of this process is the regularly oscillating level of gene activity in mesodermal tissue (Dequéant and Pourquié 2008; Oates et al. 2012). There also is a molecular gradient that travels from head to tail with constant speed-the wavefront. When it passes by cells that happen to be at a particular point of the cycle of oscillating gene activity, the wavefront defines the spatial boundary of the next segment to differentiate. Thus, segments of equal length form in a rhythmic pattern.

For an individual oscillating cell, a mechanistic model can show the genes and gene products involved, and whether they activate or inhibit one another, such as in negative feedback loops. This must be combined with a mathematical model of the complex regulatory network's functioning to explain why sustained (as opposed to damped) oscillations occur (Baker et al. 2008), why the oscillation has a particular period (which differs among vertebrate species), and why several different genes oscillate within a cell in a coordinated fashion, some in phase and others in antiphase (Goldbeter and Pourquié 2008). Across individual cells, mechanistic models account for the cell-to-cell signalling that coordinates the oscillations of adjacent cells; mathematical models account for how the oscillations across cells maintain synchrony (Herrgen et al. 2010; Morelli et al. 2009; Riedel-Kruse et al. 2007).

This case illustrates how explanations in systems biology model several levels integratively, combining interactions among molecular components of a cell (resulting in oscillations), interactions among cells (resulting in synchronization), and the interaction of oscillating tissue and the molecular wavefront (resulting in the formation of segments). Such multilevel integration is concomitant with representational selectivity. The success of these approaches depends on a judicious choice of physical entities and interactions within the system of interest, and their incorporation into models that abstract away from details or idealize features (Brigandt 2013c; Weisberg 2013). System biology’s synthesis of mathematical approaches and experimental practices expands how we understand scientific explanation. Even though philosophical accounts of mechanistic explanation were developed in contrast to law-based explanations encompassing quantitative principles, research strategies that investigate the multilevel consequences of small-scale 
molecular interactions in biological systems demonstrate how explanations must rely on a combination of causal-mechanistic and mathematical models.

\section{Representation and Manipulation in Model Systems}

Metagenomics and systems biology select boundaries that circumscribe interactions of interest in biological entities and processes (i.e., "systems"). Often, an entity or process is chosen as the basis for a more generalized understanding, and thus becomes a model system. Philosophers of science do something similar in choosing particular scientific fields on which to base their generalizations (Wimsatt 2007). We have so far emphasized cellular systems, both multitaxon communities formed by groups of cells in different environments, and intracellular networks. Additional insights from the epistemic complexities of multilevel research strategies are found in the model systems that are constituents of individual cells.

\section{Different Representations in Multilevel Research on Protein Folding} The macromolecular structures called proteins are the workhorses of cells. They function in thousands if not millions of interactive arrangements to cause replication, regeneration, transport, signalling, structural integrity and all the myriad activities that a cell must do to stay viable (Alberts et al. 2007). Proteins are constituted by sequences of amino acids that usually fold into native structures within the cells of organisms in order to perform these activities. ${ }^{3}$ The "problem" of protein folding has been characterized as predicting and explaining the actual three-dimensional structure that proteins adopt after forming linear two-dimensional amino-acid chains (Dill et al. 2008). Approaches to protein folding can be understood as an exemplar of how multilevel biological explanation works because more than just the atomic interactions of molecules constituting the protein are needed to explain their in vivo folding in the cellular environment. The explanation requires the causal contributions of folded, chaperone proteins already in three-dimensional states to assist in producing the functional conformation (Hüttemann and Love 2011; Love 2012).

Any exploration of the multilevel research strategies directed at protein folding has to pay particular attention to how diverse methodological approaches are related to one another. Three-dimensional protein folding is approached from multiple perspectives: the physics of intra-molecular forces modelled in silico, the experimental study of in vitro folding using techniques of structural chemistry, and the in vivo analysis of position, interaction and function by cell biologists (Chen et al. 2008). Each perspective provides a partial and not completely overlapping account of proteinfolding behaviour. What is learned about folding from the physics of a protein's basic components will inform but not determine what is detected from the investigation of chemical structure. Likewise, the details of chemical structure inform but do not completely specify the biological function in which the macromolecule participates.

Even for this small-scale intracellular system, we see the necessity of relating multiple perspectives to one another in order to manipulate, explain, and predict the target

\footnotetext{
${ }^{3}$ A subset of proteins is described as "intrinsically disordered" in the sense of not having a native three-dimensional conformation (Tompa 2009).
} 
behaviour. Each perspective requires input from the others to answer its research questions (Mitchell 2003, 2009). As a consequence, analyses of multilevel systems demand continual crosstalk between scientists as they investigate phenomena from different, often disciplinary-specific, perspectives. Different perspectives target different features of the same phenomenon, employ different techniques to investigate those features and express their results in different types of representations. Physicists typically explore the energetic properties of unfolded, partially folded and stably folded proteins by in silico simulations of possible conformations (e.g., Urbanc et al. 2004). Physical chemists apply nuclear magnetic resonance spectroscopy and X-ray crystallography to denatured and native protein conformations in vitro to infer spatial organization from magnetic and light diffracting behaviours (e.g., Schotte et al. 2003). Cell biologists use mutation studies and differential visualization techniques to explore interactions among multiple molecules in vivo (e.g., Walsh et al. 2002).

Philosophical attention needs to be paid to research problems and how they guide the coordinative practices that integrate these different perspectives (Brigandt 2010, 2013a; Love 2008; Mitchell 2002). Rather than a single clear-cut question with one definitive answer, problems such as protein folding are enmeshed in a wider research agenda in which multilevel strategies of inquiry articulate with additional methods, models, and fields. Specific research problems - rather than overarching theoriesstructure and make coherent the diversity of approaches employed by researchers, and facilitate systematic evaluations of the resulting contributions (Mitchell 2002; Love 2013, 2014; Brigandt and Love 2012b). For protein folding, integration among approaches occurs in numerous ways, including organized competitions in which different algorithms for protein structure prediction are tested against structures generated from experimental approaches (Moult et al. 1995; Ramsey 2007).

Philosophers have to understand how several, multilevel partial representations, which cannot simply be unified, are coordinated and used to inform one another in the context of specific problems rather than for the purpose of constructing or confirming a theory.

\section{Multilevel Manipulation of Systems as Models of Development}

We have explored multilevel systems with different, partial characterizations of spatial and temporal scales. A similar perspective is applicable to modelling strategies that move from one type of organismal organization to another, specifically from unicellularity to multicellularity. This provides a novel tool in the context of developmental biology because ontogeny is traditionally understood as a phenomenon of metazoans. However, there is a large body of research on microbial development (e.g., Brun and Shimkets 2000) and multilevel modelling of multicellular development can be accomplished with unicellular systems.

Genetics and allied molecular approaches are nowadays the standard for investigating multicellular animals and plants in developmental biology. Because the most powerful investigative techniques can be applied with greatest effect to single-celled organisms, these systems can be viewed as reductionist models of ontogeny. Many biologists assume this strategy is not viable because development involves complex multicellular dynamics not exhibited by microbes. But a closer analysis of the criteria for experimental models demonstrates that microbes can be models for some but not all developmental phenomena (Love and Travisano 2013). This is similar to the case of emergent parsimony where specific modelling objectives determine which 
theoretical simplifications may be used (Section 3). Likewise, in developmental biology, the particular explanatory aim governs when the use of single-celled experimental systems is legitimate and implies that modelling ontogeny at multiple levels with different model organisms is warranted.

These criteria for experimental models include representational similarities between source and target phenomena and mechanisms, as well as how and to what degree they can be manipulated. Preferences in applying these criteria reveal key assumptions about how model systems are used. One is that experimental models are expected to represent a variety of developmental phenomena, whereas microbes often do not. Another is that cell dynamics are conceptualized in terms of the organism ("individually"), whereas microbial interactions are described as "social”. However, moving back and forth between microbial and metazoan models with these criteria made explicit reveals the prospects and limitations for comprehending organismal ontogeny (and not just its parts) on the basis of microbial systems. This movement between different "levels" of model system is another kind of multilevel modelling (Love and Travisano 2013).

Conceptualizing “multilevel” in this way emphasizes how manipulability, in addition to representational similarity, is critical for selecting microbial molecular systems as models for evolutionary, ecological and developmental processes. Without an understanding of how microbes are manipulated as experimental tools for a range of research objectives in multilevel modelling, philosophy of science cannot account for a large swathe of successful life science. The protein-folding example above demonstrates that scientists must move back and forth between physical, chemical and biological models in order to explain and predict folding behaviour effectively, where this does not require that one of these models is more fundamental. Dynamic system modelling expands our conception of scientific investigation by exemplifying how integration across levels, rather than reduction, and coordination among various representations, rather than unification, are the governing themes.

\section{Philosophical Lessons From Multilevel Research Strategies}

We have reviewed various cases of multilevel research in molecular life sciences that have not yet received thorough investigation by philosophers, such as multitaxa microbial communities and biogeochemical systems. All of the wide-ranging approaches to diverse multilevel systems described above raise significant philosophical questions about explanation, modelling and representation, especially the demand for sophisticated integrative methods to produce new knowledge in these contexts. Deploying molecular approaches is not equivalent to embracing reductionism. Whether it is conceptualizing complex causal communities of microbes or physico-chemical systems, combining mathematical and mechanistic models, or integrating different perspectives on protein folding, the philosophical lessons all derive from various dimensions of the multilevel research strategies we have canvassed. 
The first dimension is conceptualizing the units of analysis. Multilevel representation takes into account the diversity of locally defined hierarchies-compositional and functional-because different research strategies foreground specific properties as part of the system of interest (e.g., human bodies conceptualized as ecosystems). "Levels" are not just nested spatial hierarchies but encompass heterogeneous clusters of causal properties related on different time scales that are relevant to particular inquiries. The second dimension is methodological. Different units of analysis require different combinations of methods, and these are chosen for their appropriateness to selected functional interactions that arise in the context of specific research problems. These problems coordinate different datasets about different system properties derived from multilevel methods in an ongoing manner, without an expectation that the process will bottom-out at a preferred level. The third dimension pertains to explanation. Different types of model are necessary to explain relevant features of biological systems. Depending on the data available and the research question, topdown and bottom-up approaches are employed, each of which is multilevel in its own right but involves different explanatory tactics. The focus on function and dynamics in biological systems means that causal inputs from different organizational states of the system must be included, and the hierarchies represented in these analyses are domain-specific. An important feature of this explanatory dimension is selectivity. Multilevel modelling emphasizes how aspects of a system are used as proxies to represent the larger system, or manipulations of one model system are generalized or compared to other model systems.

These three dimensions of multilevel research strategies are exemplified in contemporary molecular inquiry into biological systems. "Reduction” does not adequately describe the integrative impulse underlying this multilevel production of new biological knowledge, which goes far beyond hierarchies that correspond to welldefined levels of organization. Nor does "unification" capture how scientists relate different representations to one another, because considerable back-and-forth negotiating occurs without the presumption of deriving a canonical or primary model as the result. Molecular data and methods thus require a richer philosophical vocabulary. Moreover, local scientific aims, rather than a global unification agenda, guide the choice of representational strategies. In addition to the standard scientific representations (theories, models, and data) that philosophers have traditionally scrutinized, our discussion foregrounds the methodological strategies and explanatory aims operative in scientific practice (Love 2008, 2014). Such local aims motivate scientists to develop certain conceptualizations and guide their assessments of the fecundity of representational tools and the appropriateness of particular selectivity choices (Brigandt 2013a). An adequate analysis of contemporary biological practice-including but not limited to the fields we discussed above-must come to grips with these dimensions of multilevel research and the associated investigative strategies in order to obtain a more robust philosophical comprehension of the epistemic successes on display in molecular biological sciences.

\section{References}

Alberts, Bruce, Alexander Johnson, Julian Lewis, Martin Raff, Keith Roberts, and Peter Walter. 2007. Molecular Biology of the Cell (5th ed.). New York: Garland. 
Azam, Farooq, Tom Fenchel, John G. Field, John S. Gray, Lutz-Arend Meyer-Reil, and T. Frede Thingstad. 1983. "The Ecological Role of Water-Column Microbes in the Sea." Marine Ecology Progress Series 10:257-263.

, and Francesca Malfatti. 2007. "Microbial Structuring of Marine

Ecosystems." Nature Reviews Microbiology 5:782-791.

Baker, Ruth E., Santiago Schnell, and Philip K. Maini. 2008. "Mathematical Models for Somite Formation." In Multiscale Modeling of Developmental Systems, ed.

Santiago Schnell, Philip K. Maini, Stuart A. Newman and Timothy J. Newman, 183203. New York: Academic Press.

Bechtel, William. 2010. "The Downs and Ups of Mechanistic Research: Circadian Rhythm Research as an Exemplar." Erkenntnis 73:313-328.

78:533-557.

. 2011. "Mechanism and Biological Explanation." Philosophy of Science

. 2012. "Understanding Endogenously Active Mechanisms: A Scientific and Philosophical Challenge." European Journal for Philosophy of Science 2:233-248.

Boogerd, Fred C., Frank J. Bruggeman, Jan-Hendrik S. Hofmeyr, and Hans V. Westerhoff , eds. 2007. Systems Biology: Philosophical Foundations. Amsterdam: Elsevier.

Brigandt, Ingo. 2010. "Beyond Reduction and Pluralism: Toward an Epistemology of Explanatory Integration in Biology." Erkenntnis 73:295-311.

. 2013a. "Explanation in Biology: Reduction, Pluralism, and Explanatory Aims." Science \& Education 22:69-91.

2013b. "Integration in Biology: Philosophical Perspectives on the Dynamics of Interdisciplinarity." Studies in History and Philosophy of Biological and Biomedical Sciences 44:461-465.

. 2013c. "Systems Biology and the Integration of Mechanistic Explanation and Mathematical Explanation." Studies in History and Philosophy of Biological and Biomedical Sciences 44:477-492.

. in press. "Evolutionary Developmental Biology and the Limits of Philosophical Accounts of Mechanistic Explanation." In Explanation in Biology: An Enquiry into the Diversity of Explanatory Patterns in the Life Sciences, ed. PierreAlain Braillard and Christophe Malaterre. Berlin: Springer.

and Alan C. Love. 2012a. "Reductionism in Biology." In The

Stanford Encyclopedia of Philosophy, ed. Edward N.

Zalta.http://plato.stanford.edu/entries/reduction-biology/. 
2012b. "Conceptualizing Evolutionary Novelty: Moving Beyond

Definitional Debates." Journal of Experimental Zoology Part B (Mol Dev Evol)

318B:417-427.

Brun, Yves V., and Lawrence J. Shimkets. 2000. Prokaryotic Development.

Washington DC: ASM Press.

Burke, Catherine, Peter Steinberg, Doug Rusch, Staffan Kjelleberg, and Torsten Thomas. 2011. "Bacterial Community Assembly Based on Functional Genes Rather Than Species." Proceedings of the National Academy of Sciences USA 108:1428814293.

Chen, Yiwen, Feng Ding, Huifen Nie, Adrian W. Serohijos, Shantanu Sharma, et al. 2008. "Protein Folding: Then and Now." Archives of Biochemistry and Biophysics 469:4-19.

Craver, Carl F. 2007. Explaining the Brain: Mechanisms and the Mosaic Unity of Neuroscience. Oxford: Oxford University Press.

, and Lindley Darden. 2013. In Search of Mechanisms: Discoveries

across the Life Sciences. Chicago: University of Chicago Press.

Crawford, John W., James A. Harris, Karl Ritz, and Iain M. Young. 2005. "Towards an Evolutionary Ecology of Life in Soil." Trends in Ecology and Evolution 20:81-87.

Cryan, John F., and Timothy D. Dinan. 2012. "Mind-Altering Microorganisms: The Impact of the Gut Microbiota on Brain and Behaviour." Nature Reviews Neuroscience 13:701-712.

Darden, Lindley. 2005. "Relations among Fields: Mendelian, Cytological and Molecular Mechanisms." Studies in History and Philosophy of Biological and Biomedical Sciences 36:349-371.

DeLong, Edward F. 2009. "The Microbial Ocean From Genomes to Biomes." Nature 459:200-206.

Dequéant, Mary-Lee, and Olivier Pourquié. 2008. "Segmental Patterning of the Vertebrate Embryonic Axis." Nature Review Genetics 9:370-382.

Dethlefsen, Les, and David A. Relman. 2011. "Incomplete Recovery and Individualized Responses of the Human Distal Gut Microbiota to Repeated Antibiotic Perturbation." Proceedings of the National Academy of Sciences USA 108:4554-4561

Dill, Ken A., S. Banu Ozkan, M. Scott Shell, and Thomas R. Weiki. 2008. "The Protein Folding Problem." Annual Review of Biophysics 37:289-316.

Dinsdale, Elizabeth A, Robert A. Edwards, Dana Hall, Florent Angly, Mya Breitbart, et al. 2009. "Functional Metagenomic Profiling of Nine Biomes." Nature 452:629632. 
Edwards, Robert A., and Forest Rohwer. 2005. "Viral Metagenomics." Nature Reviews Microbiology 3:504-510.

Faith, Jeremiah J., Federico E. Rey, David O’Donnell, Maria Karlsson, Nathan P. McNulty, et al. 2011. "Creating and Characterizing Communities of Human Gut Microbes in Gnotobiotic Mice." ISME Journal 4:1094-1098.

Falkowski, Paul G., Tom Fenchel, and Edward F. DeLong. 2008. "The Microbial Engines That Drive Earth’s Biogeochemical Cycles." Science 320:1034-1039.

Faust, Karoline, and Jeroen Raes. 2012. "Microbial Interactions: From Networks to Models." Nature Reviews Microbiology 10:538-550.

Fierer, Noah, Scott Ferrenberg, Gilberto E. Flores, Antonio González, Jordan Kueneman, et al. 2012a. "From Animalcules to an Ecosystem: Application of Ecological Concepts to the Human Microbiome." Annual Review of Ecology, Evolution, and Systematics 43:137-155.

, Jonathan W. Leff, Byron J. Adams, Uffe N. Nielsen, Scott T. Bates, et al. 2012b. "Cross-Biome Metagenomic Analyses of Soil Microbial Communities and Their Functional Attributes." Proceedings of the National Academy of Sciences USA 109:21390-21395

Gilbert Jack A., Joshua A. Steele, J. Gregory Caporaso, Lars Steinbrück, Jens Reeder, et al. 2012. "Defining Seasonal Marine Microbial Community Dynamics." ISME Journal 6:298-308.

Goldbeter, Albert, and Olivier Pourquié 2008. "Modeling the Segmentation Clock as a Network of Coupled Oscillations in the Notch, Wnt and FGF Signaling Pathways." Journal of Theoretical Biology 252:574-585.

Gordon, Jeffrey I. 2012. "Honor Thy Gut Symbionts Redux." Science 336:1251-1253.

Green, Sara. 2013. "When One Model Is Not Enough: Combining Epistemic Tools in Systems Biology." Studies in History and Philosophy of Biological and Biomedical Sciences 44:170-180.

Griffiths BS, Philippot L. 2012. "Insights Into the Resistance and Resilience of the Soil Microbial Community." FEMS Microbiology Reviews 37:112-129.

Grimm, Volker. 1999. "Ten Years of Individual-Based Modelling in Ecology: What Have We Learned and What Could We Learn in the Future?" Ecological Modelling 115:129-148.

Hamady, Micah, and Rob Knight. 2009. "Microbial Community Profiling for Human Microbiome Projects: Tools, Techniques, and Challenges." Genome Research 19:1141-1152. 
Herrgen, Leah, Saúl Ares, Luis G. Morelli, Christian Schröter, Frank Jülicher, and Andrew C. Oates. 2010. "Intercellular Coupling Regulates the Period of the Segmentation Clock." Current Biology 20:1244-1253.

Hüttemann, Andreas, and Alan C. Love. 2011. "Aspects of Reductive Explanation in Biological Science: Intrinsicality, Fundamentality, and Temporality." British Journal for the Philosophy of Science 62:519-549.

Karr, Jonathan R., Jayodita C. Sanghvi, Derek N. Macklin, Miriam V. Gutschow, et al. 2012. "A Whole-Cell Computatational Model Predicts Phenotype from Genotype." Cell 150:389-401.

Klipp, Edda, Wolfram Liebermeister, Christoph Wierling, Axel Kowald, Hans Lehrach, and Ralf Herwig. 2010. Systems Biology: A Textbook. Weinheim: Wiley$\mathrm{VCH}$.

Klitgord, Niels, and Daniel Segrè. 2011. "Ecosystems Biology of Microbial Metabolism." Current Opinion in Biotechnology 22:541-546.

Krohs, Ulrich, and Werner Callebaut. 2007. "Data without Models Merging with Models without Data." In Systems Biology: Philosophical Foundations, ed. Fred C. Boogerd, Frank J. Bruggeman, Jan-Hendrik S. Hofmeyr, and Hans V. Westerhoff, 181-213. Amsterdam: Elsevier.

Larsen, Peter E., Yuki Hamada, and Jack Gilbert. 2012. "Modeling Microbial Communities: Current, Developing and Future Technologies for Predicting Microbial Community Interaction." Journal of Biotechnology 160:17-24.

, Frank R. Collart, Dawn Field, Folker Meyer, Christopher S. Henry, et al. 2011. "Predicted Relative Metabolic Turnover (PRMT): Determining Metabolic Turnover From a Coastal Marine Metagenomic Dataset." Microbial Informatics and Experimentation 1:4.

Ley, Ruth E., Daniel A. Peterson, and Jeffrey I. Gordon. 2006. "Ecological and Evolutionary Forces Shaping Microbial Diversity in the Human Intestine." Cell 124:837-848.

Lindeman, Raymond L. 1942. "The Trophic Dynamic Aspect of Ecology." Ecology 23: $399-417$

Love, Alan C. 2008. "Explaining Evolutionary Innovation and Novelty: Criteria of Explanatory Adequacy and Epistemological Prerequisites." Philosophy of Science 75:874-886.

. 2012. "Hierarchy, Causation and Explanation: Ubiquity, Locality and Pluralism." Interface Focus 2:115-125.

. 2013. "Interdisciplinary Lessons for the Teaching of Biology from the Practice of Evo-Devo." Science \& Education 22:255-278. 
2014. The erotetic organization of development. In Towards a Theory of Development, eds. A. Minelli and T. Pradeu, 33-55. Oxford: Oxford University Press.

and Michael Travisano. 2013. "Microbes Modeling Ontogeny."

Biology \& Philosophy 28:161-188.

McDonald, Daniel, Yoshiki Vazquez-Baeza, William A. Walters, J. Gregory

Caporaso, and Rob Knight. 2013. "From Molecules to Dynamic Biological

Communities." Biology \& Philosophy 28: 241-259.

Mitchell, Sandra D. 2002. "Integrative Pluralism." Biology \& Philosophy 17:55-70.

2003. Biological Complexity and Integrative Pluralism. Cambridge:

Cambridge University Press.

2009. Unsimple Truths: Science, Complexity and Policy. Chicago:

University of Chicago Press.

Morelli, Luis G., Saúl Ares, Leah Herrgen, Christian Schröter, Frank Jülicher, and Andrew C. Oates. 2009. "Delayed Coupling Theory of Vertebrate Segmentation." HFSP Journal 3:55-66.

Moult, John, Jan T. Pedersen, Richard Judson, and Krzysztof Fidelis. 1995. "A LargeScale Experiment to Assess Protein Structure Prediction Methods." Proteins:

Structure, Function, and Bioinformatics 23:ii-iv.

Oates, Andrew C., Luis G. Morelli, and Saúl Ares. 2012. "Patterning Embryos with Oscillations: Structure, Function and Dynamics of the Vertebrate Segmentation Clock." Development 139:625-639.

O’Malley, Maureen A., and Orkun S. Soyer. 2012. "The Roles of Integration in Molecular Systems Biology." Studies in History and Philosophy of Biological and Biomedical Sciences 43:58-68.

Philippot, Laurent, Siv G. E. Andersson, Tom J. Battin, James I. Prosser, Joshua P. Schimel, et al. 2010. "The Ecological Coherence of High Bacterial Taxonomic Ranks." Nature Reviews Microbiology 8:523-529.

Pomeroy, Lawrence R. 1974. "The Ocean’s Food Web, a Changing Paradigm." BioScience 24:499-504.

Raes, Jeroen, and Peer Bork. 2008. "Molecular Eco-Systems Biology: Towards an Understanding of Community Function.” Nature Reviews Microbiology 6:693-699.

Railsback, Steven F. 2001. "Concepts From Complex Adaptive Systems as a Framework for Individual-Based Modelling." Ecological Modelling 139:47-62.

Ramsey, Jeffry L. 2007. "Calibrating and Constructing Models of Protein Folding." Synthese 155:307-320. 
Riedel-Kruse, Ingmar H., Claudia Müller, and Andrew C. Oates. 2007. "Synchrony Dynamics During Initiation, Failure, and Rescue of the Segmentation Clock." Science 317:1911-1915.

Riesenfeld, Christian S., Patrick D. Schloss, and Jo Handelsman. 2004.

"Metagenomics: Genomic Analysis of Microbial Communities." Annual Review of Genetics 38:525-552.

Rohwer, Forest, and Katie Barott. 2013. "Viral Information." Biology \& Philosophy 28:283-297.

and Rebecca V. Thurber. 2009. "Viruses Manipulate the Marine

Environment." Nature 459:207-212.

Rondon, Michelle R., Paul R. August, Alan D. Bettermann, Sean F. Brady, Trudy H. Grossman, et al. 2000. "Cloning the Soil Metagenome: A Strategy For Accessing the Genetic and Functional Diversity of Uncultured Microorganisms." Applied and Environmental Microbiology 66:2541-2547.

Schotte, Friedrich, Manho Lim, Timothy A. Jackson, Aleksandr V. Smirnov, Jayashree Soman, et al. 2003. "Watching a Protein as it Functions with 150-ps TimeResolved X-Ray Crystallography." Science 300:1944-1947.

Shade, Ashley, and Jo Handelsman. 2012. "Beyond the Venn Diagram: The Hunt For a Core Microbiome." Environmental Microbiology 14:4-12.

Smith, Michelle I., Tanya Yatsunenko, Mark J. Manary, Indi Trehan, Rajhab Mkakosya, et al. 2013. "Gut Microbiomes of Malawian Twin Pairs Discordant for Kwashiorkor." Science 339:548-554.

Sterelny, Kim. 2006. "Local Ecological Communities." Philosophy of Science 73:215231.

Suttle, Curtis A. 2005. "Marine Viruses - Major Players in the Global Ecosystem." Nature Reviews Microbiology 5:801-812.

Tompa, P. 2009. Structure and Function of Disordered Proteins. New York: CRC Press.

Turnbaugh, Peter J., and Jeffrey I. Gordon. 2008. "An Invitation to the Marriage of Metagenomics and Metabolomics." Cell 134:708-713.

Micah Hamady, Tanya Yatsunenko, Brandi L. Cantarel, Alexis

Duncan, et al. 2009. "A Core Gut Microbiome in Obese and Lean Twins." Nature 457:480-484.

Tyson, Gene W., Jarrod Chapman, Philip Hugenholtz, Eric E. Allen, Rachna J. Ram, et al. 2004. "Community Structure and Metabolism Through Reconstruction of Microbial Genomes From the Environment." Nature 428:37-43. 
Urbanc, Brigita, Luis Cruz, Sijung Yun, Sergey V. Buldyrev, Gal Bitan, et al. 2004. "In Silico Study of Amyloid ß-Protein Folding and Oligomerization." Proceedings of the National Academy of Sciences USA 101:17345-17350.

Ursell, Luke K, Jose C. Clemente, Jai R. Rideout, Dirk Gevers, J. Gregory Caporaso, and Rob Knight. 2012. "The Interpersonal and Intrapersonal Diversity of HumanAssociated Microbiota in Key Body Sites." Journal of Allergy and Clinical Immunology 129:1204-1208.

VerBerkmoes, Nathan C., Vincent J. Denef, Robert L. Hettich, and Jillian F. Banfield. 2009. "Systems Biology: Functional Analysis of Natural Microbial Consortia Using Community Proteomics." Nature Reviews Microbiology 7:196-205.

Walsh, Dominic M., Igor Klyubin, Julia V. Fadeeva, William K. Cullen, Roger Anwyl, et al. 2002. "Naturally Secreted Oligomers of Amyloid ß Protein Potently Inhibit Hippocampal Long-Term Potentiation In Vivo." Nature 416:535-539.

Weisberg, M. (2013). Simulation and Similarity: Using Models to Understand the World. New York: Oxford University Press.

Wimsatt, William C. 2007. Re-Engineering Philosophy for Limited Beings: Piecewise Approximations to Reality. Cambridge, MA: Harvard University Press.

Young, Iain M., John W. Crawford, Naoise Nunan, Wilfred Otten, and Andrew Spiers. 2008. "Microbial Distribution in Soils: Physics and Scaling." Advances in Agronomy 100:81-121.

Zarraonaindia, Iratxe, Daniel P. Smith, and Jack A. Gilbert. 2013. "Beyond the Genome: Community-Level Analysis of the Microbial World." Biology \& Philosophy 28:261-182.

Zengler, Karsten, and B. O. Palsson. 2012. "A Road Map for the Development of Community Systems (CoSy) Biology." Nature Reviews Microbiology 10:366-372. 